\title{
Evaluation of Treatment for Childhood Medulloblastoma, with Special Reference to Combination Therapy with Interferon and Radiation
}

\author{
Kenro SunAmi, Akimasa Uozumi, Akio SEMBA, \\ Akira YAMAURA, Hiroyasu MAKINO and Takeyoshi MIYOSHI* \\ Departments of Neurological Surgery and \\ *Radiology, School of Medicine, Chiba University, Chiba
}

\begin{abstract}
Thirteen children, aged 6 months to 14 years, underwent surgery for medulloblastoma at Chiba University Hospital from 1971 to 1985 . The nine patients treated prior to 1980 received postoperative irradiation therapy with or without chemotherapy. Since 1980, four patients have been treated postoperatively with interferon (IFN) plus radiation therapy. The IFN was administered intramuscularly once or twice a week throughout the period of radiation therapy.

Six of the nine patients not treated with IFN died within 1 year. In this group, the 3-and 5-year survival rates were $33 \%$ and $22 \%$, respectively. One of the four patients treated with IFN died within 10 months of surgery, and another is disabled. The remaining two patients are in good condition. The 3and 5-year survival rates in the IFN group were both 75\% as of the last follow-up in January of 1986.
\end{abstract}

Key words: medulloblastoma, interferon, radiation therapy, combination therapy

\section{Introduction}

Medulloblastoma accounts for $20-30 \%$ of all intracranial tumors in children. ${ }^{1,4)}$ It is considered a highly malignant tumor because of its early recurrence and intraspinal metastasis. Many authors believe that radiation therapy is, at the present time, the most reliable treatment for this tumor, but the poor prognosis even with irradiation demands more effective therapy. ${ }^{22)}$ Clinical trials of interferon (IFN) for malignant intracranial tumors have increased in number, but so far its effectiveness as a single therapy is reportedly only $20-30 \%,{ }^{19,20)}$ which is about the same as that of other chemotherapies. Since 1980, the authors have treated medulloblastoma with a postoperative combination of IFN and irradiation, and our results have been encouraging.

\section{Patients and Methods}

We treated 13 children with medulloblastoma, aged 6
Received
February 12, 1986;
Accepted November

months to 14 years, at Chiba University Hospital from 1971 to 1985 . All underwent tumor resection through suboccipital craniectomy. The diagnosis of cerebellar medulloblastoma was confirmed in all cases by histological examination of surgical specimens. Our principle of treatment was to remove as much of the tumor as possible and then to irradiate the whole neuroaxis, with at least 4500 rads applied to the posterior fossa. Before 1980 nine patients received radiation therapy with or without chemotherapy; since 1980 four patients have been given IFN and irradiation. The patients' characteristics and their treatments are summarized in Table 1.

We used human leukocyte IFN, type $\alpha$, induced by HVJ (Sendai virus), which produces high titers of crude IFN. The crude IFN was purified by a modification of Cantell's method. ${ }^{2,18)}$ The specific activity of the IFN was $6 \times 10^{6}$ to $6 \times 10^{7}$ units $/ \mathrm{mg}$ protein. The IFN, $250 \times 10^{4}$ to $500 \times 10^{4}$ units per day, was administered intramuscularly once or twice at the end of each week throughout the period of radiation therapy (Table 2). These treatments were started 1 week after operation. The last follow-up was conducted in January of 1986. 
Table I Cases of cerebellar medulloblastoma in children

\begin{tabular}{|c|c|c|c|c|c|c|c|c|}
\hline \multirow{2}{*}{$\begin{array}{l}\text { Case } \\
\text { No. }\end{array}$} & \multirow{2}{*}{ Age } & \multirow{2}{*}{ Sex } & \multirow{2}{*}{$\begin{array}{l}\text { Type of } \\
\text { operation }\end{array}$} & \multicolumn{3}{|c|}{ Radiation } & \multirow{2}{*}{ Survival time } & \multirow{2}{*}{ Outcome } \\
\hline & & & & Whole brain & Posterior fossa & Whole spine & & \\
\hline 1 & 3 yrs & $\mathbf{M}$ & partial & & & & $1 \mathrm{mo}$ & dead \\
\hline 2 & 2 yrs & $F$ & partial & 2800 & 3800 & & $9 \mathrm{mos}$ & dead \\
\hline 3 & $1 \mathrm{yr}$ & $F$ & biopsy & 1000 & 4500 & 2500 & $7 \operatorname{mos}$ & dead \\
\hline 4 & $6 \mathrm{mos}$ & $\mathbf{M}$ & subtotal & & 5060 & & $9 \operatorname{mos}$ & dead \\
\hline 5 & $1 \mathrm{yr}$ & $\mathrm{F}$ & subtotal & 3000 & 5000 & & 11 yrs 3 mos & alive \\
\hline 6 & 8 yrs & $\mathbf{M}$ & partial & 5060 & & 2970 & 3 yrs 4 mos & dead \\
\hline 7 & 14 yrs & $F$ & partial & 3840 & 5280 & 3050 & $6 \mathrm{mos}$ & dead \\
\hline 8 & 5 yrs & $\mathrm{F}$ & subtotal & 4120 & & 2520 & $5 \operatorname{mos}$ & dead \\
\hline 9 & 8 yrs & $\mathbf{M}$ & subtotal & 4540 & & 2560 & $6 \mathrm{yrs} 11 \mathrm{mos}$ & alive \\
\hline $10^{*}$ & 5 yrs & $\mathrm{F}$ & subtotal & 2990 & 4490 & 2400 & $4 \mathrm{yrs} 11 \mathrm{mos}$ & alive \\
\hline $11^{*}$ & $4 \mathrm{yrs}$ & $\mathrm{M}$ & partial & 3140 & 5240 & 2500 & 4 yrs 9 mos & alive \\
\hline $12^{*}$ & $12 \mathrm{yrs}$ & $\mathbf{M}$ & subtotal & 4540 & 5940 & 5000 & 3 yrs 6 mos & alive \\
\hline $13^{*}$ & $5 \mathrm{yrs}$ & $\mathbf{M}$ & partial & 3000 & 4600 & 5000 & $10 \operatorname{mos}$ & dead \\
\hline
\end{tabular}

*Treated with IFN.

Table 2 Summary of patients treated with IFN

\begin{tabular}{cccc}
\hline Case & \multicolumn{2}{c}{ IFN- $\alpha$ dose $($ IU) } & Performance \\
\cline { 2 - 3 } No. & Weekly & Total & status $(\%)^{*}$ \\
-10 & $5 \times 10^{6} \times 2$ & $1.75 \times 10^{8}$ & 90 \\
11 & $5 \times 10^{6} \times 2$ & $1.30 \times 10^{8}$ & 100 \\
12 & $5 \times 10^{6} \times 2$ & $1.55 \times 10^{8}$ & 30 \\
13 & $2.5 \times 10^{6} \times 1$ & $3.75 \times 10^{7}$ & - \\
\hline${ }^{*}$ Karnofsky et al. ${ }^{(0)}$ & &
\end{tabular}

\section{Results}

As shown in Tables 1 and 2, Cases from 1 to 9 were treated up to 1979 without IFN, and Cases from 10 to 13 were treated with IFN since 1980.

The postoperative courses of the nine patients treated without IFN are shown in Fig. 1. Six died within 1 year. There are only two survivors: 11 years, 3 months and 6 years, 11 months. These patients are free of disease and enjoy a useful life. The longest follow-up period was 11 years, 3 months. The 3 - and 5 -year survival rates were $33 \%$ and $22 \%$, respectively (Fig. 2).

One of the four patients treated with combined IFN and radiation therapy died 10 months after craniectomy (Fig. 3). The remaining three are alive at 3.5 to almost 5 years postoperatively. Two are in good condition and one is bedridden. The tumor metastasized to the left frontal tip in one survivor and to the frontal base in another. The 3- and 5-year survival rates are both $75 \%$, according to the criteria of Culter et al. ${ }^{3)}$ (Fig. 2).

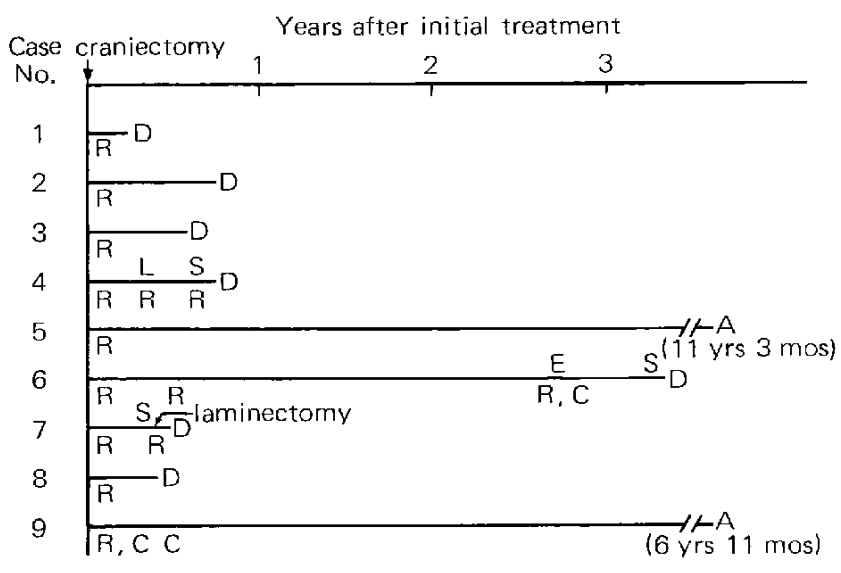

Fig. 1 Postoperative courses of medulloblastoma in children treated before 1980 with surgery and radiation therapy, with or without chemotherapy. $\mathrm{R}$ : radiation, $\mathrm{C}$ : chemotherapy, $\mathrm{L}$ : local recurrence, $\mathrm{S}$ : spinal cord metastasis, $\mathrm{E}$ : extracranial metastasis, D: dead, A: alive.

\section{Case Reports}

Case 10: A 5-year-old female underwent subtotal removal of a medulloblastoma, followed by irradiation and IFN therapy. The IFN was injected intramuscularly in a total dose of $1.75 \times 10^{8}$ units. Remission persisted for 2 years, 7 months, when a recurrence was detected on the left frontal tip. The metastatic tumor was removed and radiation therapy was administered postoperatively. At 4 years, 11 months after her initial surgery, she is healthy and is attending school. 


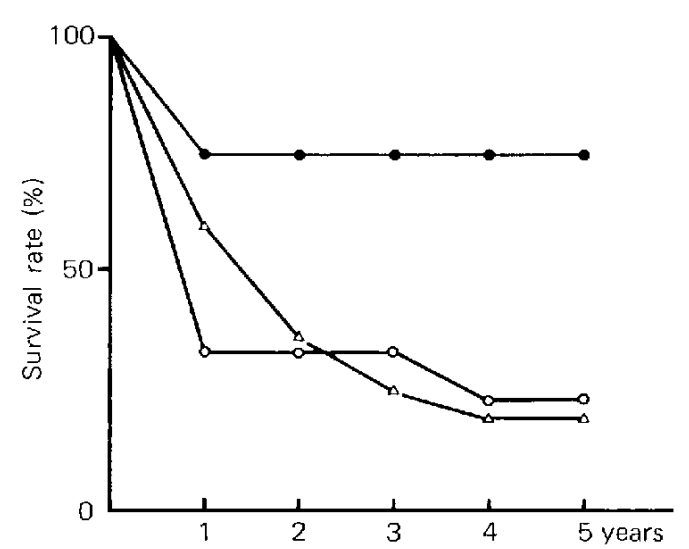

Fig. 2 Survival rate of medulloblastoma in children. $O$ : treated with surgery plus radiation therapy, with or without chemotherapy (1971 to 1979). : treated with IFN in combination with surgery and radiation therapy (1980 to 1985). $\triangle$ : National Survey conducted in Japan in 1982 .

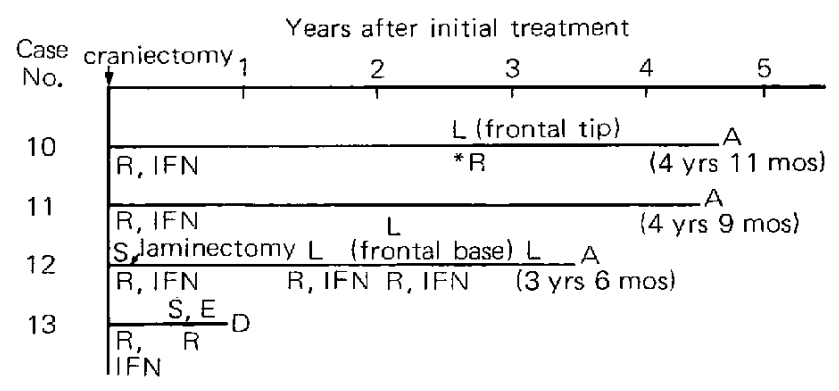

Fig. 3 Postoperative courses of medulloblastoma in children treated since 1980 with surgery followed by IFN in combination with radiation therapy. *Frontal lobectomy.

Case 11: Radiation therapy and IFN, in a total dose of $1.30 \times 10^{8}$ units, were given to a 4-year-old male following partial removal of a medulloblastoma. Four years, 9 months later, he is free of tumor and doing well.

Case 12: A 12-year-old male already had two metastatic lesions in a cerebellar hemisphere at the time of surgery for the original tumor. Postoperatively he received irradiation to the whole brain (4540 rads), followed by local irradiation. A spinal metastasis was also found at the $T h_{12}-\mathrm{L}_{2}$ level; it was decompressed by laminectomy and irradiated with 5000 rads. IFN was administered 31 times in a total dose of $1.55 \times 10^{8}$ units. The patient was in remission for 16 months, until a recurrence was detected. The new tumor was treated with irradiation and IFN. A few months later, a frontal base metastasis was found (Fig. 4), and this was also treated with irradiation and IFN. During the most recent follow-up period, he was hospitalized to treat another local regrowth.

Case 13: A metastatic lesion in the spinal cord was noticed at the time of diagnosis of cerebellar medulloblastoma in a 5-year-old male. Postoperatively, a total of $3.75 \times 10^{7}$ units of IFN was given in conjunction with irradiation to the whole neuroaxis. After a 9-month remission, a new metastasis was observed in the sacral region. This patient died 1 month after the recurrence despite retreatment with irradiation.

\section{Discussion}

Medulloblastoma is regarded as highly malignant because of its early recurrence and high frequency of spinal metastasis. Although irradiation is believed to be the most effective therapy for this tumor, 6 years ago we found, on reviewing the medulloblastoma cases managed in our hospital, that the outcome of such treatment is very poor. For that reason, in 1980 we introduced IFN therapy. In this report we compare the results of therapy for medulloblastoma before and after IFN was added to our treatment regimen.

There have been several reports of brain tumors treated with IFN alone. ${ }^{7,19,20)}$ Although the experimental work can be seen in the literature, ${ }^{11.17)}$ as far as we know, only our group has reported on combination therapy with IFN and irradiation in clinical cases of medulloblastoma. ${ }^{15,16)}$

Six of the nine patients not treated with IFN died within 1 year of initial surgery. The 3- and 5-year survival rates were $33 \%$ and $22 \%$, respectively. These
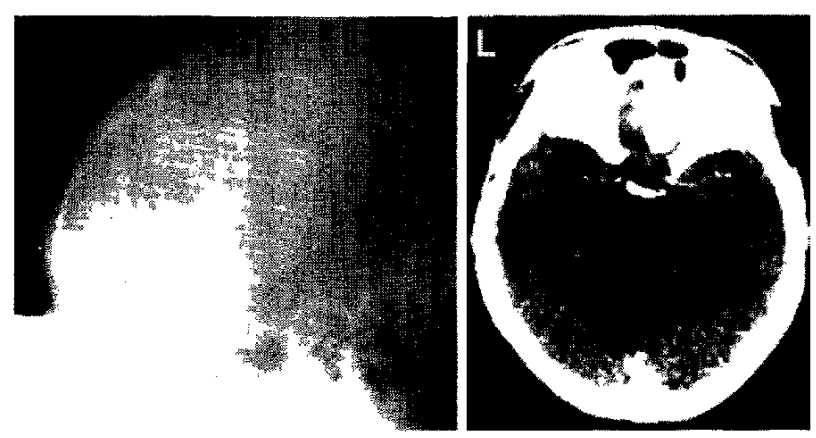

Fig. 4 Case 12. left: A linac-graphy. Note that the cribriform plate was covered with an eye shield. right: $\mathrm{CT}$ scan showing metastasis to the frontal base. 
results are about the same as those of the $1982 \mathrm{Na}$ tional Survey in Japan. Odake et al. ${ }^{22)}$ reviewed 13 cases of medulloblastoma and concluded that the poor outcomes were partly due to inadequate radiation therapy, which did not adhere to the general principle that the whole neuroaxis must be irradiated and in which doses were not high enough. We found that a total irradiation dose of 4500 to 5000 rads to the posterior fossa was not administered in some of our cases. In other cases, the spinal cord was not irradiated. We attribute the differences in treatment partly to differences in the patients' physical condition and age and partly to differences of opinion among the surgeons.

Of the four patients to whom we administered IFN along with irradiation, only one died within 1 year of treatment. Of the remaining three survivors, two had metastases. We have speculated the reason for metastasis as follows; some areas had not been irradiated although we believed that the whole neuroaxis had been irradiated. One patient had a metastasis to the cribriform plate, which was concave at the midline and had been blocked by an eye shield during irradiation (Fig. 4). Another had a metastasis to the frontal tip, which apparently had shifted out of the irradiation field because of the strict localization of the field. These sites are easily missed if special attention is not paid to them during irradiation. This pitfall, which has been noted by others ${ }^{9,12)}$ as well, was not recognized until computerized tomographic scanning (CT scan) became available. Therefore, the irradiation should be applied to the entire central nervous system; no area should be missed.

Some authors ${ }^{6,21,25)}$ believe that only extensive removal of the tumor yields a good prognosis, while others ${ }^{12,13)}$ contend that surgery does not improve the prognosis. Our own results were equivocal. Among the patients treated before 1980 , two of the four who underwent subtotal (macroscopic) tumor removal are still alive and functioning well. Both of the children subjected to subtotal removal after 1980 are alive, although only one is in good condition. Obviously, we cannot draw conclusions from these results, because the patient population was too small.

Treatment for malignant brain tumors with IFN$\alpha^{7,20)}$ and $1 \mathrm{FN}-\beta^{14,19,24)}$ alone has been reported. Hirakawa et al. ${ }^{\text {? }}$ stated that partial remission (clinical or radiological improvement) was obtained in two of 10 malignant brain tumor cases with IFN- $\alpha$. Nagai ${ }^{19)}$ reported that IFN- $\beta$ was effective (more than $50 \%$ reduction of tumor size by CT scan) in $22.2 \%$ of their malignant brain tumor series. The results are almost the same with IFN- $\alpha$ and IFN- $\beta$. No significant differences in efficacy between system- ic and local administration have been noted, although local injection has been known to cause meningitis. ${ }^{20)}$ IFN does not appear to be a particularly promising agent for therapy of malignant brain tumors when used alone. " Hirakawa et al. ${ }^{7}$ reported two cases of medulloblastoma among their brain tumor series. Although one experienced a partial response to IFN, the other showed no improvement, and both patients died. Nagai ${ }^{19}$ reported that among the seven cases of medulloblastoma in his series of 54 malignant brain tumors, a complete response (tumor disappearance by CT scan) was obtained in two with IFN monotherapy.

A precise clinical regimen for combination therapy with IFN and irradiation has not yet been established, primarily because it is not clear whether IFN confers a cytoprotective effect or augments the efficacy of irradiation. Our laboratory studies ${ }^{17)}$ clearly indicated that the suppression of growth by irradiation was not enhanced when sarcoma 180 cells were pretreated with IFN. Pretreatment with IFN apparently reduced the effect of irradiation on these cells, or showed radioprotective activity. The growth suppression was significantly enhanced when IFN was treated after irradiation. Ito $\left.{ }^{8}\right)$ observed similar effects with IFN. Talas et al. ${ }^{26)}$ reported that the survival of mice was prolonged when radiation was administered after an IFN inducer was given, when the serum IFN level was at its highest. These investigators also found radioprotective activity by IFN in endogenous spleen colony formation tests. IFN is thought to inhibit cell proliferation by preventing the cells from entering the $G_{1}-S$ phase, ${ }^{5,23)}$ which prevents DNA synthesis and thereby provides a radioprotective effect. On the other hand, when IFN was administered after irradiation, the cells damaged by irradiation, especially those cells on the verge of death, are apt to receive inhibition of DNA synthesis by IFN, by which suppression of cell growth is enhanced. If IFN likewise alters the cell cycle in vivo, it is reasonable to irradiate prior to IFN administration. Accordingly, we administered IFN at the end of each week. However, the method of administration of IFN needs to be studied in further detail. The necessary amount of IFN dosage and irradiation are also required to be investigated.

In conclusion, our findings suggest that in the treatment of medulloblastoma, combination therapy with IFN and irradiation may be superior to treatment without IFN. Further study is needed to determine the most effective treatment regimen. 


\section{References}

1) Bloom HJG, Wallace ENK, Henk JM: The treatment and prognosis of medulloblastoma in children. Amer $J$ Roentgen 105: 43-62, 1969

2) Cantell K, Hirvonen S: Large-scale production of human leukocyte interferon containing $10^{8}$ units per ml. J Gen Virol 39: 541-543, 1978

3) Culter SJ, Ederer F: Maximum utilization of the life table method in analyzing survival. J Chronic Dis 8: 699-712, 1958

4) Farwell JR, Dohrmann GJ, Flannery JT: Central nervous system tumors in children. Cancer 40: 31233132,1977

5) Fuse A, Kuwata T: Effects of interferon on the human clonal cell line, RSa inhibition of macromolecular synthesis. J Gen Virol 33: 17-24, 1976

6) Gerosa MA, Di Stefano E, Olivi A, Carteri A: Multidisciplinary treatment of medulloblastoma. A 5-year experience with the S10P trial. Childs Brain 8: $107-118,1981$

7) Hirakawa $K$, Ueda $S$, Nakagawa $Y$, Suzuki $K$, Fukuma S: Effect of human leukocyte interferon on malignant brain tumors. Cancer 51: 1976-1981, 1983

8) Ito $\mathbf{H}$ : Antitumor and radiation sensitizing effects of human leukocyte interferon in vitro. Nippon Igaku Hoshasen Gakkai Zasshi 41: 551-558, 1981 (in Japanese)

9) Jerb B, Krishnaswami S, Reid A, Allen JC: Radiation for medulloblastoma adjusted to prevent recurrence to the cribriform plate region. Cancer 54: 602604,1984

10) Karnofsky DA, Abelmann WH, Craver LF, Burchenal JH: The use of the nitrogen mustards in the palliative treatment of carcinoma. Cancer 1: 634656,1948

11) Korosue K, Tamaki N, Matsumoto S, Takeshita I, Fukui $\mathrm{M}$ : Interferon-modification of the in vitro radiation response in cultured human KNS-42 cells. Neurol Med Chir (Tokyo) 24: 233-239, 1984 (in Japanese)

12) Koshino $\mathrm{K}$, Abekura $\mathrm{S}$, Mizuta $\mathrm{T}$, Mogami $\mathrm{H}$ : Follow-up study of 171 infantile medulloblastoma on operative effectiveness. Nerv Syst Children 9: 303308, 1984 (in Japanese)

13) Mealey JJ, Hall PV: Medulloblastoma in children; survival and treatment. I Neurosurg 46: 56-64, 1977

14) Miki Y: Anti-neoplastic effect of human fibroblast interferon on human malignant gliomas transplanted into nude mice and its clinical application. Neurol Med Chir (Tokyo) 22: 785-796, 1982 (in Japanese)

15) Miyoshi T, Saito M, Arimizu N: Effect of interferon $($ IFN- $\alpha$ ) combined with radiation therapy on human malignant tumors, in: Proceeding of the 41st Annual Meeting of the Japanese Cancer Association. 1982, p 387 (in Japanese)

16) Miyoshi $T$, Saito $M$, Arimizu $N$, Makino $H$, Yamaura A, Kono M, Senba A, Shin H, Nishiyama H, Akiyama S, Yanagihara F: Preliminary study of children's malignant brain tumors with interferon $(\mathrm{IFN}-\alpha)$ and radiation, in Kishida T (ed): Proceedings of the International Symposium on Interferons. 1983, pp 420-430

17) Miyoshi T, Saito M, Arimizu N, Yanagihara F, Akiyama S: Modifying effects of interferon (IFN) on the growth of irradiated sarcoma 180 cells in vitro. Nippon Igaku Hoshasen Gakkai Zasshi 44: 88-92, 1984 (in Japanese)

18) Mogensen KE, Cantell K: Production and preparation of human leukocyte interferon. Pharmacol Ther [C] 1: 369-381, 1977

19) Nagai $M$ : Clinical trials of human fibroblast interferon (HuIFN- $\beta$ ) on malignant brain tumors. Nippon Gan Chiryo Gakkai Shi 18: 60-68, 1983 (in Japanese)

20) Nakagawa $Y$ : Interferon therapy for primary brain tumors (Part 1). Local administration. Neurol Med Chir (Tokyo) 24: 83-89, 1984 (in Japanese)

21) Norris OG, Bruce DA, Byrd RL, Schut L, Littman P, Bilaniuk L, Zimmerman RA, Capp R: Improved relapse-free survival in medulloblastoma utilizing modern techniques. Neurosurgery 9: 661-664, 1981

22) Odake G, Suzuki K, Naruse S, Yamaki T, Fujimoto M, Horikawa $Y$, Oota $T$, Toyama $M$, Hirakawa K: Treatment and prognosis of medulloblastoma. A study of 13 surgical cases. Neurol Med Chir (Tokyo) 20: 1123-1130, 1980 (in Japanese)

23) O'Shaugnessy MV, Lee SHS, Rozee KR: Interferon inhibition of DNA synthesis and cell division. Canad J Microbiol 18: 145-151, 1972

24) Otsuka $S$, Handa $H$, Yamashita $J$, Suda K, Takeuchi $\mathrm{J}$ : Single agent therapy of interferon for brain tumors. Correlation between natural killer activity and clinical course. Acta Neurochir (Wien) 73: 1323,1984

25) Raimondi AJ, Tomita T: Medulloblastoma in childhood. Comparative results of partial and total resection. Childs Brain 5: 310-328, 1979

26) Talas $M$, Szolgay $E$ : Radioprotective activity of interferon inducers. Arch Virol 56: 309-315, 1978

Address reprint requests to: K. Sunami, M.D., Department of Neurological Surgery, School of Medicine, Chiba University, 1-8-1 Inohana, Chiba 280, Japan. 